\title{
A!
}

This is an electronic reprint of the original article.

This reprint may differ from the original in pagination and typographic detail.

Poutanen, Mikko; Ahmed, Zafar; Rautkari, Lauri; Ikkala, Olli; Priimägi, Arri

\section{Thermal Isomerization of Hydroxyazobenzenes as a Platform for Vapor Sensing}

Published in:

ACS Macro Letters

DOI:

10.1021/acsmacrolett.8b00093

Published: 20/03/2018

Document Version

Publisher's PDF, also known as Version of record

Published under the following license:

CC BY-NC

Please cite the original version:

Poutanen, M., Ahmed, Z., Rautkari, L., Ikkala, O., \& Priimägi, A. (2018). Thermal Isomerization of Hydroxyazobenzenes as a Platform for Vapor Sensing. ACS Macro Letters, 7(3), 381-386.

https://doi.org/10.1021/acsmacrolett.8b00093

This material is protected by copyright and other intellectual property rights, and duplication or sale of all or part of any of the repository collections is not permitted, except that material may be duplicated by you for your research use or educational purposes in electronic or print form. You must obtain permission for any other use. Electronic or print copies may not be offered, whether for sale or otherwise to anyone who is not an authorised user. 


\title{
Thermal Isomerization of Hydroxyazobenzenes as a Platform for Vapor Sensing
}

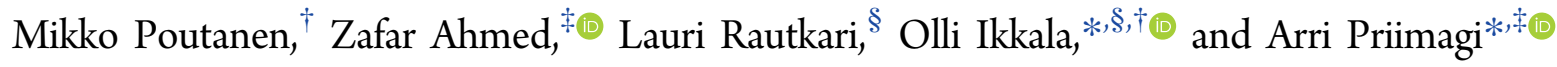 \\ ${ }^{\dagger}$ Department of Applied Physics, Aalto University, P.O. Box 15100, FI-00076, Aalto, Espoo, Finland \\ ${ }^{\ddagger}$ Laboratory of Chemistry and Bioengineering, Tampere University of Technology, P.O. Box 541, FI-33101, Tampere, Finland \\ ${ }^{\S}$ Department of Bioproducts and Biosystems, Aalto University, P.O. Box 16300, FI-00076, Aalto, Espoo, Finland
}

\section{Supporting Information}

ABSTRACT: Photoisomerization of azobenzene derivatives is a versatile tool for devising light-responsive materials for a broad range of applications in photonics, robotics, microfabrication, and biomaterials science. Some applications rely on fast isomerization kinetics, while for others, bistable azobenzenes are preferred. However, solid-state materials where the isomerization kinetics depends on the environmental conditions have been largely overlooked. Herein, an approach to utilize the environmental sensitivity of isomerization kinetics is developed. It is demonstrated that thin polymer films containing hydroxyazobenzenes offer a conceptually novel platform for sensing hydrogen-bonding vapors in the environment. The
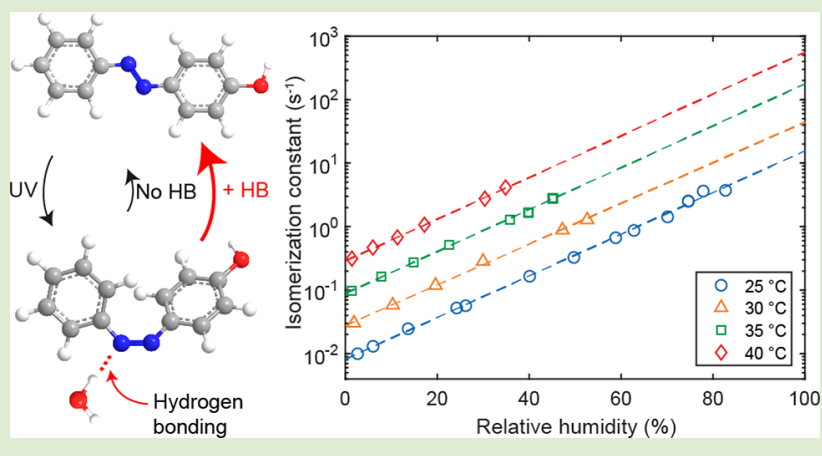
concept is based on accelerating the thermal cis-trans isomerization rate through hydrogen-bond-catalyzed changes in the thermal isomerization pathway, which allows for devising a relative humidity sensor with high sensitivity and quick response to relative humidity changes. The approach is also applicable for detecting other hydrogen-bonding vapors such as methanol and ethanol. Employing isomerization kinetics of azobenzenes for vapor sensing opens new intriguing possibilities for using azobenzene molecules in the future.

$\mathrm{P}$ hotoswitchable and photochromic compounds provide a unique platform for devising stimuli-responsive materials whose function and properties can be remotely manipulated with light, with high spatiotemporal resolution. ${ }^{1}$ Their light sensitivity stems from photoinduced changes in molecular conformation and electronic properties, which in recent years have been extensively utilized in applications ranging from optical memories ${ }^{2,3}$ and light-to-mechanical energy conversion, ${ }^{4,5}$ to photocontrol of chemical reactions ${ }^{6}$ and biological functions. ${ }^{7,8}$ The utility of photoswitchable materials can be further expanded if the materials are multiresponsive, that is, their optical response can be tuned via changes in environmental conditions (e.g., humidity, $\mathrm{pH}$, presence of analytes). This offers a conceptual basis for photochromic sensors, ${ }^{9,10}$ which have been developed from diarylethenes and spiropyrans, for example, for sensing anions, ${ }^{11}$ amines, ${ }^{12}$ and thiols. ${ }^{13}$ For azobenzenes, to the best of our knowledge, such an isomerization kinetics based concept has not been demonstrated.

Azobenzenes are a particularly versatile class of photoswitchable compounds, as they exhibit two isomeric states, trans and cis, with a large difference in geometry, absorption spectra, and dipole moment. The power of azobenzenes lies in the fact that the lifetime of the metastable cis-isomer can be controlled over a wide range, from milliseconds in push-pull azobenzenes $^{14}$ up to even several months in ortho-substituted azobenzenes. ${ }^{15}$ While fast thermal relaxation is useful in, for example, optical switching ${ }^{16}$ and the long-lived cis-state desired in photobiology, ${ }^{17}$ azobenzene derivatives whose thermal isomerization dynamics depends strongly on the environmental conditions are particularly interesting. As the most prominent example, 4-hydroxyazobenzenes can experience a change of up to 5 orders of magnitude in the cis-lifetime in nonpolar versus polar solvents due to hydrogen-bond-assisted tautomerization. ${ }^{14,18}$ The photochemistry of hydroxyazobenzene derivatives has been comprehensively studied in solutions. ${ }^{19-21}$ In the solid state, they have been utilized as building blocks for supramolecular self-assemblies ${ }^{22-24}$ and photoactive units in light-responsive polymer systems. ${ }^{25}$ However, none of these studies makes use of the huge "dynamic range" in the isomerization kinetics of hydroxyazobenzenes, which has been largely disregarded in the solid state.

Herein, we show that the isomerization kinetics of 4hydroxyazobenzenes in a polymeric environment offers an excellent, conceptually novel platform for sensing hydrogenbonding vapors. More precisely, we devise and characterize a relative humidity sensor, which is fast, reliable, and accurate. We also demonstrate sensitivity to other hydrogen bonding

Received: February 2, 2018

Accepted: March 5, 2018

Published: March 10, 2018 
a<smiles>CCc1ccc(N=Nc2ccc(O)cc2)cc1</smiles>

C

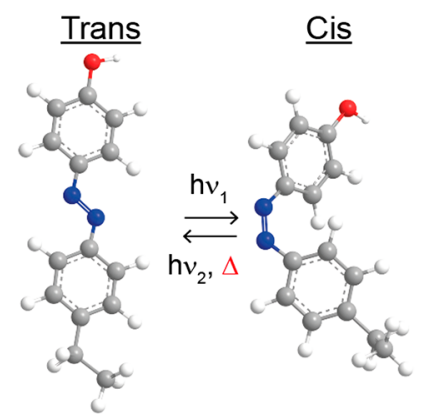

e

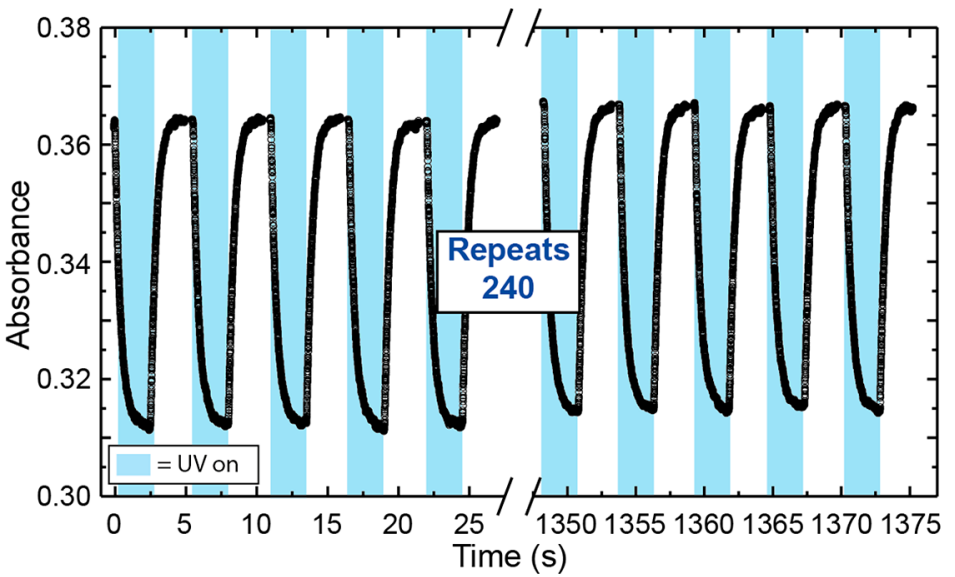

d

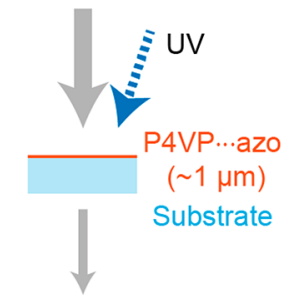

Photodiode

(spectrophotometer) b

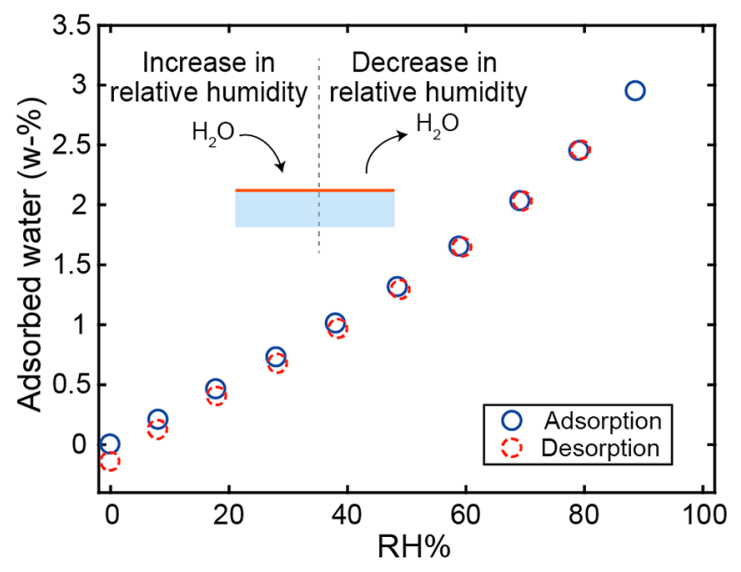

Figure 1. (a) Supramolecular, nominally stoichiometric complex of poly(4-vinylpyridine) (P4VP) and 4-(4-ethylphenylazo)phenol (2PAP). (b) Spectral changes of a thin film of nominally equimolar P4VP(2PAP) complex after UV irradiation. Inset: thermal cis-trans relaxation of the same sample. (c) Trans- and cis-isomers of 2PAP. (d) Simplified schematic representation of the experimental geometry. The gray and blue arrows indicate white probe light and UV pump light, respectively. (e) An example of the reproducibility of the thermal isomerization over 250 pumping-relaxation cycles. (f) Adsorption and desorption isotherms of water at $25^{\circ} \mathrm{C}$ for the equimolar P4VP(2PAP) complex.

vapors, such as methanol and ethanol, and outline the possibility for monitoring several hydrogen-bonding vapors, or vapor content and temperature, simultaneously. We believe to propose a completely new way of using azobenzenecontaining photoresponsive polymers for sensing vapors.

Relative humidity ( $\mathrm{RH}$ ) is generally measured either by capacitive or resistive sensors, and a large variety of different sensors having electrical or optical readouts have been built. $^{26-28}$ The majority of the sensors are based on adsorption of water molecules from the gaseous environment to an active sensing material where it changes permittivity or conductivity or induces swelling, translating into the readout signal. These sensors often measure an extrinsic property of the sensory material, which is dependent on the exact geometry, that is, needs device-specific calibration. In contrast, our sensor concept relies on isomerization kinetics, which is an intrinsic material property. Therefore, the exact geometry is irrelevant and no device-specific calibration is needed, which is a significant benefit compared to the commercial devices.

As depicted in Figure 1a, we use 4-(4-ethylphenylazo)phenol (2PAP) embedded into a solid poly(4-vinylpyridine) (P4VP) matrix. P4VP and 2PAP form hydrogen-bonded supramolecular complexes, allowing high 2PAP loading to be used without phase separation: thin films of $\mathrm{P} 4 \mathrm{VP}(2 \mathrm{PAP})$ remain amorphous and of high optical quality, even at a nominally equimolar complexation ratio, corresponding to $80 \mathrm{wt} \%$ azobenzene concentration. ${ }^{29}$ Upon UV ( $365 \mathrm{~nm}$ ) irradiation, the 2PAP molecules undergo efficient trans-cis isomerization in the solid state, as illustrated by the spectral changes shown in Figure $1 \mathrm{~b}$ (Figure 1c displays the trans- and cis-isomers of 2PAP). The samples are thin films $(\sim 1 \mu \mathrm{m})$ on glass/quartz substrates and the spectral changes are measured in transmission (Figure 1d). The spectral changes are typical to azobenzene derivatives: the $\pi-\pi^{*}$ transition centered at $352 \mathrm{~nm}$ decreases significantly upon UV illumination, while the $n-\pi^{*}$ transition at $450 \mathrm{~nm}$ strengthens, indicating efficient trans-cis isomerization.

The inset of Figure $1 \mathrm{~b}$ displays the thermal cis-trans isomerization of the 2PAP molecules in the P4VP matrix. In liquids and in polymers above glass transition temperature $\left(T_{\mathrm{g}}\right)$, the thermal isomerization typically follows simple first-order kinetics. However, it turns out that a stretched exponential function, that is, Kohlrausch-Williams-Watts function, explains better our findings:

$$
A(t)=\left(A_{0}-A_{\infty}\right) e^{-(k t)^{\beta}}+A_{\infty}
$$

where $A_{0}$ is the absorbance after illumination, $A_{\infty}$ is the absorbance of the fully relaxed, that is, all-trans, state, $\beta$ is the stretching exponent, and $k$ is the rate constant of thermal isomerization. In fact, this is not surprising, as the stretched exponential function is known to explain, for example, glassy 

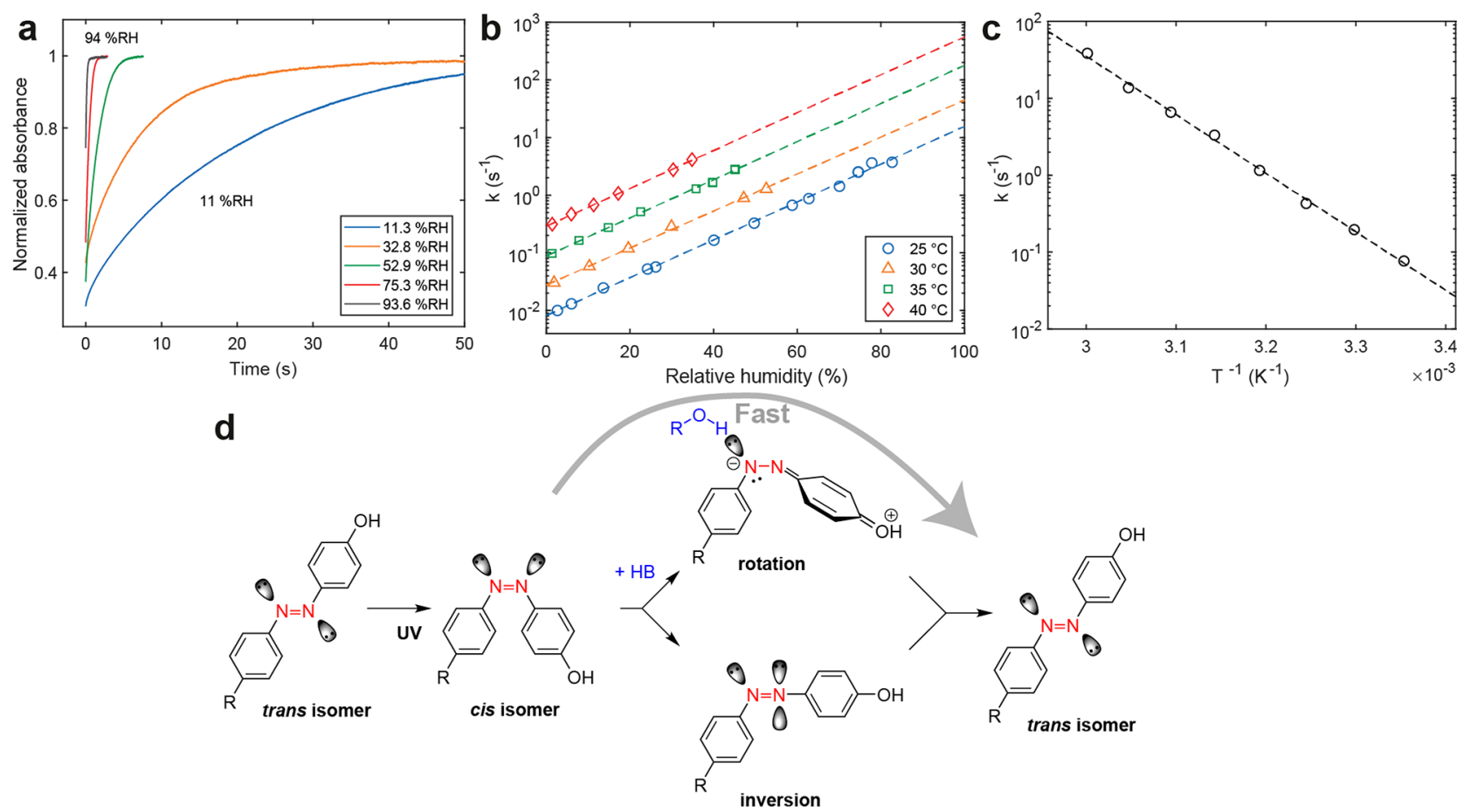

Figure 2. Relative-humidity dependence of thermal isomerization of a thin film of nominally equimolar P4VP(2PAP) complex. (a) Thermal isomerization curves at different RH values. (b) Rate constants of the cis-trans isomerization at different $\mathrm{RH}$ values at different temperatures. (c) Arrhenius-type temperature dependence of the rate constant of thermal isomerization measured at $30 \% \mathrm{RH}$. (d) Proposed mechanism for the sensitivity of the isomerization rate constant to the presence of hydrogen-bonding species.

relaxations. ${ }^{30}$ The effect of using stretching exponential function is minimal for our results and we retain from further analysis of the stretching exponents. In our material system, the isomerization process is highly reproducible, as illustrated in Figure 1e: no changes in isomerization dynamics were observed over 250 subsequent repeat cycles (the standard deviation of the fitted time constants was $2.7 \%$ ), provided that the experimental conditions (temperature, humidity) remain unchanged. Even if the azobenzene units may eventually degrade by photo-oxidation, they show potential to withstand over at least 20000 repeat cycles without significant degradation ( $<5 \%$ absorbance decrease).

The vapor-sensing ability of the $\mathrm{P} 4 \mathrm{VP}(2 \mathrm{PAP})$ complexes is based on the slight hydrophilicity of the material. Due to this, even if both P4VP and 2PAP are insoluble in water, their complex is able to adsorb a small amount of water (or other hydrogen-bonding molecules) from the environment. As shown in Figure 1f, the water vapor uptake is approximately $3.0 \mathrm{wt} \%$ at $90 \% \mathrm{RH}$, which translates to about 0.6 water molecules per 2PAP molecule for the equimolar complex. In general, more molecules will adsorb upon an increase in $\mathrm{RH}$ and vice versa. The shape of the adsorption isotherm depends on the chemical activity of the vapor species as well as the properties of the polymer material, and it is important for the sensing ability.

The thermal isomerization rate constant, $k$, shows a strong exponential dependence $\left(\sim e^{\lambda \cdot \mathrm{RH}}\right)$ on the relative humidity, $\mathrm{RH}$ (Figure 2a,b), and the sample-related constant, $\lambda$, is $0.0755 \pm$ $0.0021 \%^{-1}$. The rate constants at $25{ }^{\circ} \mathrm{C}$ vary from 0.01 to 3.73 $\mathrm{s}^{-1}$ between $2.6 \%$ and $82.7 \%$ relative humidity. The change is nearly 400-fold, and the exponential behavior suggests the difference between $0 \% \mathrm{RH}$ and $100 \% \mathrm{RH}$ to be 1000 -fold for the equimolar complex. This yields a huge dynamic range for the isomerization-based humidity sensor. Measurements at differ- ent temperatures reveal the Arrhenius-type $\left(\sim e^{-E_{a} / R T}\right)$ temperature dependence (Figure2c) of the rate constant, with apparent energy of activation, $E_{a}$ of $146 \pm 7 \mathrm{kcal} / \mathrm{mol}$. The dependence on $\mathrm{RH}$ is exponential at all the studied temperatures (Figure 2b). This shows that the $\mathrm{RH}$ and temperature dependencies are decoupled, which is an important feature from the sensing point of view. Overall, based on the results of Figure $2 a-c$, the time constant can be described as

$$
k(T, R H)=k_{0} e^{-E_{\mathrm{a}} / R T} \cdot e^{\lambda \cdot \mathrm{RH}}
$$

where $k_{0}$ is a sample-dependent constant, $E_{\mathrm{a}}$ is the apparent energy of activation, $R$ is the gas constant, and $\mathrm{RH}$ is the relative humidity at temperature $T$.

We believe that the RH dependence arises from the waterinduced intrinsic changes in the isomerization pathway. The hypothesized mechanism is depicted in Figure 2d. The two classical routes for thermal cis-trans isomerization are inversion and rotation. ${ }^{14}$ For an unsubstituted azobenzene molecule, inversion is favored, while for push-pull type azobenzenes, rotation is the dominating isomerization pathway. The latter has a significantly lower activation energy, resulting in shorter lifetimes of the cis-isomer. In 4-hydroxyazobenzenes, hydrogenbonding to the $-\mathrm{N}=\mathrm{N}-$ moiety by, for example, water or ethanol molecules shifts the azo-hydrazone equilibrium toward the hydrazone form, thus, effectively easing the rotational isomerization pathway. ${ }^{31,32}$ This leads to a drastic decrease in the cis-lifetimes, that is, an increase in the thermal isomerization rate constants upon increasing $\mathrm{RH}$. The lack of side reactions allows the isomerization cycles to be repeated continuously and with high accuracy, which renders hydroxyazobenzenes optimal for devising a reliable, high-performance sensor for water vapor or other hydrogen-bonding vapors. 

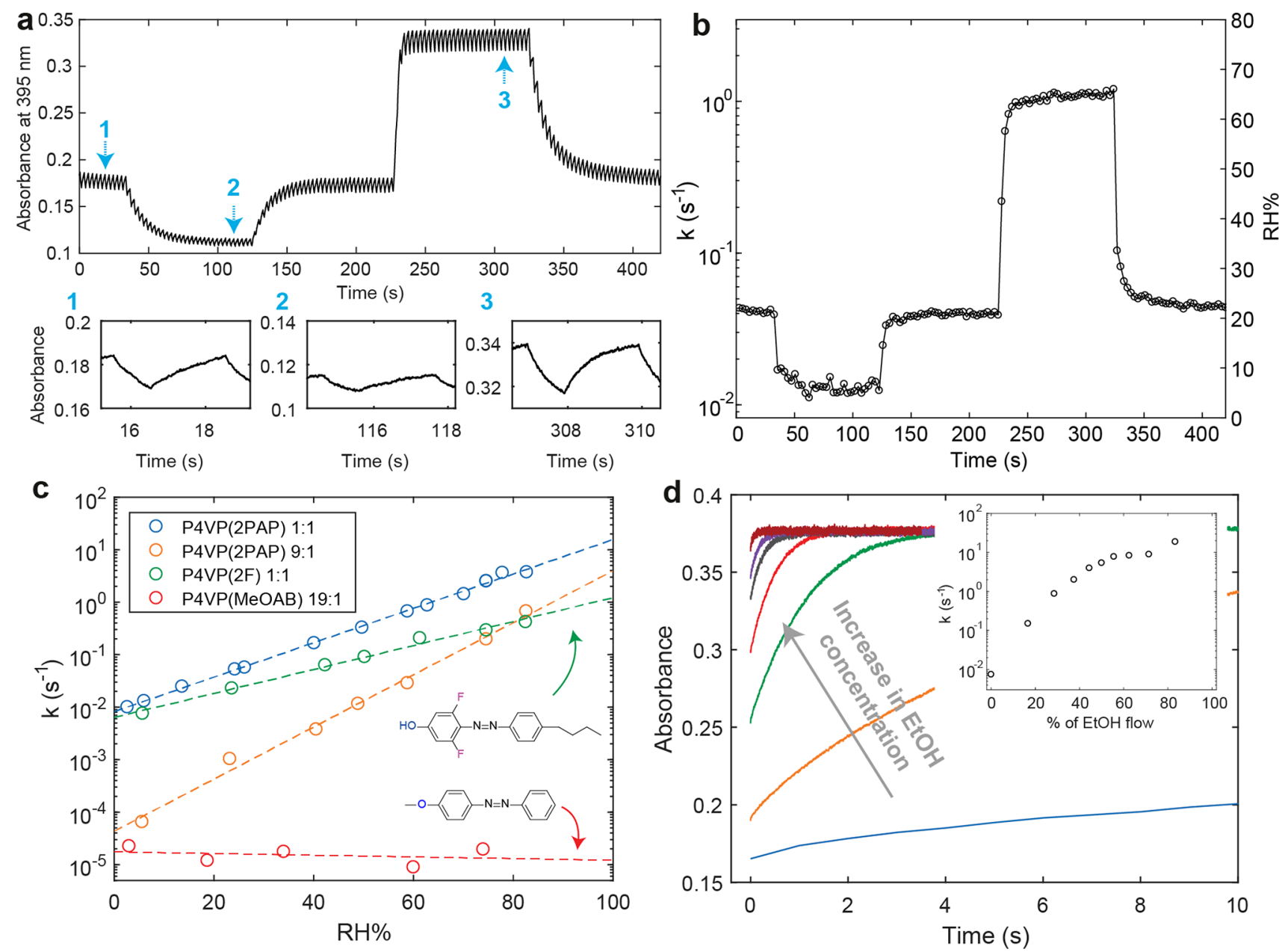

Figure 3. (a) Example of the use of the isomerization kinetics as a relative humidity sensor by pulsed UV irradiation ( $1 \mathrm{~s})$ and following the cis-trans isomerization (2 s). The insets 1-3 show examples of the partial thermal relaxations (increase in absorbance) at three different $\mathrm{RH}$ values $(\sim 20 \%$ $\mathrm{RH}, \sim 5 \% \mathrm{RH}$, and $\sim 70 \% \mathrm{RH}$, respectively). (b) Rate constants extracted from the relaxations in (a) and the RH values obtained from eq 2 and data shown in Figure 2. (c) RH dependencies of rate constants for different azobenzene-containing polymer systems. (d) Sensitivity of the rate constants to the presence of ethanol. The inset shows the rate constants as a function of percentage of ethanol flow $\left(\mathrm{N}_{2}\right.$ bubbled through ethanol).

To demonstrate the potential of the proposed method in an actual sensing device, the sample is kept constantly in a nonequilibrium state by pulsed illumination with $365 \mathrm{~nm}$ light, and the kinetics of the thermal isomerization is measured for each relaxation. Upon humidity transients from $\sim 20 \% \mathrm{RH}$ to low $(\sim 5 \% \mathrm{RH})$ and high humidities $(\sim 70 \% \mathrm{RH})$, the thermal isomerization constant changes rapidly. Based on the exponential dependence, the logarithmic scale of the rate constants is translated to linear scale of $\mathrm{RH}$ values (Figure $3 \mathrm{~b}$ ). A comparison to a commercial hygrometer is shown in the Supporting Information, Figure S1. Upon an increase of humidity from $20 \% \mathrm{RH}$ to $70 \% \mathrm{RH}$, the response time for $90 \%$ change is $11 \mathrm{~s}$ and upon decrease is $15 \mathrm{~s}$. The response speed is a result of the fast equilibration of the adsorption of water molecules into the polymer film, arising from the use of a thin (ca. $1 \mu \mathrm{m}$ ) active layer.

Based on our results, the hydroxyazobenzene-based vapor sensor is fast, accurate, and reliable. We note that the use of hydroxyazobenzenes is critical for it to function. This is demonstrated in Figure $3 \mathrm{c}$ by using $5 \mathrm{~mol} \%$ mixtures (with respect to monomers) of 4-methoxyazobenzene (MeOAB) and P4VP. The methoxy functionality is not able to tautomerize as the hydroxy group is, and therefore, the mixture does not show any dependence on the RH. Furthermore, in higher mixing ratios, the $\mathrm{MeOAB}$ phase separates from the polymer, unlike the supramolecular $\mathrm{P} 4 \mathrm{VP}(2 \mathrm{PAP})$ complex where the phenolpyridine hydrogen bonding allows high azobenzene concentration to be used. Figure $3 \mathrm{c}$ also shows that the sensitivity of humidity detection can be optimized by changing the material composition. Using $10 \mathrm{~mol} \%$ of $2 \mathrm{PAP}$ in P4VP (see Figure S2 for spectra), instead of the equimolar complexation, increases the hydrophilicity of the material system and decreases the cislifetime at dry conditions, ${ }^{29}$ leading to a dynamic range of 5 orders of magnitude. On the other hand, when 2PAP is replaced with its ortho-fluorinated counterpart, 4-((4butylphenyl)diazenyl)-3,5-difluorophenol (2F) (inset of Figure 3c; see Figure S2 for the absorption spectrum), the material becomes more hydrophobic and the hydrogen-bond-accepting ability of the cis-azo group weakens. Both of these decrease the $\mathrm{RH}$ sensitivity, as is clearly evident from Figure 3c. Overall, these results confirm the proposed mechanism, that is, that 4hydroxyazobenzene derivatives are able to create functional sensors, the major requirement being a tautomerizable azobenzene molecule and a matrix that does not contain hydrogen-bond donors. Finally, Figure $3 \mathrm{~d}$ demonstrates that the sensing ability is not limited to water, but the system can detect also other hydrogen-bonding vapors, as demonstrated here using ethanol. In principle, by combining different 
hydroxyazobenzene molecules with distinct spectral changes into the same matrix, one can foresee the possibility of simultaneously measuring relative humidity and temperature or independently detecting several hydrogen-bonding vapors within one photoactive polymer film.

In summary, we have shown that the thermal isomerization kinetics of 4-hydroxyazobenzenes offer a robust platform for sensing hydrogen-bonding vapors. The strong dependence on the environment, that is, the high sensitivity of the device demonstrated, arises from the intrinsic changes in the isomerization pathway through tautomerization, induced by the presence of hydrogen-bond-donating molecules such as water vapor. The dependence of the thermal lifetime on relative humidity is shown to be exponential, and changes of up to 5 orders of magnitude between dry and wet conditions were observed. Importantly, the Arrhenius-type temperature dependence of thermal isomerization is decoupled from the dependence on relative humidity close to room temperature. The reproducibility of the isomerization allows using the isomerization kinetics as an optically readable vapor sensor with high accuracy. In addition to relative humidity, the sensor can detect also other hydrogen bonding vapors, as demonstrated for ethanol. The sensitivity of the device can be optimized by tuning the material composition. Importantly, the concept provides a pathway toward remote sensing of gases and vapors using fiber-optic connections and suppresses the need for device-specific calibration taken the type of the gas or vapor is known. We present here what we believe to be the first azobenzene isomerization-kinetics-based vapor sensor, yet are confident that by clever materials design, the concept can be expanded toward other types of analytes as well.

\section{EXPERIMENTAL SECTION}

Materials and Methods. Poly(4-vinylpyridine) $\left(M_{\mathrm{n}}=1000 \mathrm{~g} /\right.$ mol) was purchased from Polymer Source and solvents were purchased from Sigma-Aldrich and used as received. The 4-(4Ethylphenylazo)phenol was synthesized through azo-coupling of 4ethylaniline and phenol according to a previously published procedure, $^{33}$ and 4-methoxyazobenzene (purity > 99.0\%) was purchased from Sigma-Aldrich and used as such. (E)-4-((4Butylphenyl)diazenyl)-3,5-difluorophenol was synthesized through azo-coupling of 3,5-difluorophenol and diazonium salt of butyl aniline (see details from Supporting Information). Thin films were prepared by dissolving poly(4-vinylpyridine) (P4VP) and the azobenzene in question into chloroform $(15 \mathrm{mg} / \mathrm{mL})$ and mixing them to obtain the desired molar ratio. The solutions were spin coated onto glass or quartz substrates with spinning conditions chosen such that the maximum absorbance of the films would be around unity. The UV-vis absorption spectra of the thin films under dark conditions were measured using Agilent Cary 5000 spectrophotometer. The spectral change upon thermal isomerization kinetics was measured using an Ocean Optics 2000+ diode array spectrometer with a deuteriumhalogen light source (DH-2000 BAL, Ocean Optics).

Thermal Isomerization Measurements. The cis-lifetimes were determined by following the absorbance at a single wavelength (395 or $340 \mathrm{~nm}$ ) by using either Agilent Cary 60 spectrophotometer or a photodiode equipped with a $10 \mathrm{~nm}$ bandpass filter $(398 \mathrm{~nm}$, OD 4, Edmund Optics). A $365 \mathrm{~nm}$ light-emitting diode (Thorlabs) equipped with a $10 \mathrm{~nm}$ bandpass filter was used to induce the trans-cis isomerization. The intensity and duration of the illumination was controlled electronically. To avoid any unwanted isomerization, the probe beam was incident on the sample only when collecting the data and blocked otherwise. The maximum observed absorbance change at $395 \mathrm{~nm}$ was $70 \%$, but the actual absorbance change is limited by the thermal rate constant (temperature, gaseous environment) and illumination intensity. Stretched exponential function (eq 1) is used for the fitting, except in cases of partial relaxations measured upon sensing, for example, Figure 3 a.

Relative Humidity Control. The isomerization measurements were done inside a temperature and humidity-controlled chamber equipped with glass windows for transmission measurements. The temperature was controlled with a resistive heating element and a Eurotherm temperature controller. The relative humidity was controlled by combining controlled flows of dry nitrogen with a flow bubbled through water. The temperature and relative humidity in the measurement chamber was measured with TESTO 635-2 hygrometer.

Dynamic Vapor Sorption. The water vapor adsorption of the equimolar complex $\mathrm{P} 4 \mathrm{VP}(2 \mathrm{PAP})$ was measured by dynamic vapor sorption equipment DVS ET (Surface Measurement Systems). The sorption cycle was $0-90 \% \mathrm{RH}$ with $10 \% \mathrm{RH}$ steps and desorption cycle employing a reverse sequence. The sample was measured as a drop-casted thin film on a thin glass substrate. The weight of the substrate was subtracted from the results.

\section{ASSOCIATED CONTENT}

\section{Supporting Information}

The Supporting Information is available free of charge on the ACS Publications website at DOI: 10.1021/acsmacrolett.8b00093.

Supporting figures and synthesis details (PDF).

\section{AUTHOR INFORMATION}

\section{Corresponding Authors}

*E-mail arri.priimagi@tut.fi.

*E-mail olli.ikkala@aalto.fi.

\section{ORCID}

Zafar Ahmed: 0000-0002-4737-6238

Olli Ikkala: 0000-0002-0470-1889

Arri Priimagi: 0000-0002-5945-9671

\section{Author Contributions}

The manuscript was written through contributions of all authors. All authors have given approval to the final version of the manuscript.

\section{Notes}

The authors declare no competing financial interest.

\section{ACKNOWLEDGMENTS}

This work was supported by the Academy of Finland through Center of Excellence of Molecular Engineering of Biosynthetic Hybrid Materials (HYBER; Decision No. 272361) and the Academy Research Fellowship Program (Decision Nos. 277091 and 284553), as well as by the European Research Council (Starting Grant Project PHOTOTUNE; Agreement No. 679646), the financial support of which A.P. gratefully acknowledges.

\section{REFERENCES}

(1) Russew, M.-M.; Hecht, S. Photoswitches: From Molecules to Materials. Adv. Mater. 2010, 22, 3348-3360.

(2) Klajn, R.; Wesson, P. J.; Bishop, K. J. M.; Grzybowski, B. A. Writing Self-Erasing Images Using Metastable Nanoparticle Inks. Angew. Chem., Int. Ed. 2009, 48, 7035-7039.

(3) Shishido, A. Rewritable Holograms Based on AzobenzeneContaining Liquid-Crystalline Polymers. Polym. J. 2010, 42, 525-533.

(4) Photomechanical Materials, Composites and Systems: Wireless Transduction of Light into Work; White, T. J., Ed.; John Wiley and Sons, 2017. 
(5) Kularatne, R. S.; Kim, H.; Boothby, J. M.; Ware, T. H. Liquid Crystal Elastomer Actuators: Synthesis, Alignment, and Applications. J. Polym. Sci., Part B: Polym. Phys. 2017, 55, 395-411.

(6) Stoll, R. S.; Hecht, S. Artificial Light-Gated Catalyst Systems. Angew. Chem., Int. Ed. 2010, 49, 5054-5075.

(7) Beharry, A. A.; Woolley, G. A. Azobenzene Photoswitches for Biomolecules. Chem. Soc. Rev. 2011, 40, 4422-4437.

(8) Szymański, W.; Beierle, J. M.; Kistemaker, H. A. V.; Velema, W. A.; Feringa, B. L. Reversible Photocontrol of Biological Systems by the Incorporation of Molecular Photoswitches. Chem. Rev. 2013, 113, 6114-6178.

(9) Qin, M.; Huang, Y.; Li, F.; Song, Y. Photochromic Sensors: A Versatile Approach for Recognition and Discrimination. J. Mater. Chem. C 2015, 3, 9265-9275.

(10) Pu, S.-Z.; Sun, Q.; Fan, C.-B.; Wang, R.-J.; Liu, G. Recent Advances in Diarylethene-Based Multi-Responsive Molecular Switches. J. Mater. Chem. C 2016, 4, 3075-3093.

(11) Xie, X.; Mistlberger, G.; Bakker, E. Reversible Photodynamic Chloride-Selective Sensor Based on Photochromic Spiropyran. J. Am. Chem. Soc. 2012, 134, 16929-16932.

(12) Valderrey, V.; Bonasera, A.; Fredrich, S.; Hecht, S. LightActivated Sensitive Probes for Amine Detection. Angew. Chem., Int. Ed. 2017, 56, 1914-1918.

(13) Shiraishi, Y.; Yamamoto, K.; Sumiya, S.; Hirai, T. Spiropyran as a Reusable Chemosensor for Selective Colorimetric Detection of Aromatic Thiols. Phys. Chem. Chem. Phys. 2014, 16, 12137-12142.

(14) Bandara, H. M. D.; Burdette, S. C. Photoisomerization in Different Classes of Azobenzene. Chem. Soc. Rev. 2012, 41, 18091825.

(15) Bléger, D.; Schwarz, J.; Brouwer, A. M.; Hecht, S. O -Fluoroazobenzenes as Readily Synthesized Photoswitches Offering Nearly Quantitative Two-Way Isomerization with Visible Light. J. Am. Chem. Soc. 2012, 134, 20597-20600.

(16) Hrozhyk, U. A.; Serak, S. V.; Tabiryan, N. V.; Hoke, L.; Steeves, D. M.; Kimball, B. R. Azobenzene Liquid Crystalline Materials for Efficient Optical Switching with Pulsed And/or Continuous Wave Laser Beams. Opt. Express 2010, 18, 8697-8704.

(17) Dong, M.; Babalhavaeji, A.; Samanta, S.; Beharry, A. A.; Woolley, G. A. Red-Shifting Azobenzene Photoswitches for in Vivo Use. Acc. Chem. Res. 2015, 48, 2662-2670.

(18) Garcia-Amorós, J.; Velasco, D. Understanding the Fast Thermal Isomerisation of Azophenols in Glassy and Liquid-Crystalline Polymers. Phys. Chem. Chem. Phys. 2014, 16, 3108.

(19) Kojima, M.; Nebashi, S.; Ogawa, K.; Kurita, N. Effect of Solvent on Cis-to-Trans Isomerization of 4-Hydroxyazobenzene Aggregated through Intermolecular Hydrogen Bonds. J. Phys. Org. Chem. 2005, 18, 994-1000.

(20) Dunn, N. J.; Humphries, W. H.; Offenbacher, A. R.; King, T. L.; Gray, J. A. pH-Dependent Cis $\rightarrow$ Trans Isomerization Rates for Azobenzene Dyes in Aqueous Solution. J. Phys. Chem. A 2009, 113, 13144-13151.

(21) Garcia-Amorós, J.; Sánchez-Ferrer, A.; Massad, W. a.; Nonell, S.; Velasco, D. Kinetic Study of the Fast Thermal Cis-to-Trans Isomerisation of Para-, Ortho- and Polyhydroxyazobenzenes. Phys. Chem. Chem. Phys. 2010, 12, 13238-13242.

(22) de Wit, J.; van Ekenstein, G. A.; Polushkin, E.; Kvashnina, K.; Bras, W.; Ikkala, O.; ten Brinke, G. Self-Assembled Poly(4-vinylpyridine)-Surfactant Systems Using Alkyl and Alkoxy Phenylazophenols. Macromolecules 2008, 41, 4200-4204.

(23) Hiekkataipale, P.; Löbling, T. I.; Poutanen, M.; Priimagi, A.; Abetz, V.; Ikkala, O.; Gröschel, A. H. Controlling the Shape of Janus Nanostructures through Supramolecular Modification of ABC Terpolymer Bulk Morphologies. Polymer 2016, 107, 456-465.

(24) Zhao, Y.; Thorkelsson, K.; Mastroianni, A. J.; Schilling, T.; Luther, J. M.; Rancatore, B. J.; Matsunaga, K.; Jinnai, H.; Wu, Y.; Poulsen, D. Small-Molecule-Directed Nanoparticle Assembly towards Stimuli-Responsive Nanocomposites. Nat. Mater. 2009, 8, 979-985.

(25) Priimagi, A.; Vapaavuori, J.; Rodriguez, F. J.; Faul, C. F. J.; Heino, M. T.; Ikkala, O.; Kauranen, M.; Kaivola, M. Hydrogen-
Bonded Polymer-Azobenzene Complexes: Enhanced Photoinduced Birefringence with High Temporal Stability through Interplay of Intermolecular Interactions. Chem. Mater. 2008, 20, 6358-6363.

(26) Farahani, H.; Wagiran, R.; Hamidon, M. Humidity Sensors Principle, Mechanism, and Fabrication Technologies: A Comprehensive Review. Sensors 2014, 14, 7881-7939.

(27) Chen, Z.; Lu, C. Humidity Sensors: A Review of Materials and Mechanisms. Sens. Lett. 2005, 3, 274-295.

(28) Yeo, T. L.; Sun, T.; Grattan, K. T. V. Fibre-Optic Sensor Technologies for Humidity and Moisture Measurement. Sens. Actuators, A 2008, 144, 280-295.

(29) Poutanen, M.; Ikkala, O.; Priimagi, A. Structurally Controlled Dynamics in Azobenzene-Based Supramolecular Self-Assemblies in Solid State. Macromolecules 2016, 49, 4095-4101.

(30) Palmer, R. G.; Stein, D. L.; Abrahams, E.; Anderson, P. W. Models of Hierarchically Constrained Dynamics for Glassy Relaxation. Phys. Rev. Lett. 1984, 53, 958-961.

(31) Asano, T.; Okada, T. Thermal Z-E Isomerization of Azobenzenes. The Pressure, Solvent, and Substituent Effects. J. Org. Chem. 1984, 49, 4387-4391.

(32) Dokić, J.; Gothe, M.; Wirth, J.; Peters, M. V.; Schwarz, J.; Hecht, S.; Saalfrank, P. Quantum Chemical Investigation of Thermal Cis-toTrans Isomerization of Azobenzene Derivatives: Substituent Effects, Solvent Effects, and Comparison to Experimental Data. J. Phys. Chem. A 2009, 113, 6763-6773.

(33) Suwanprasop, S.; Suksorn, S.; Nhujak, T.; Roengsumran, S.; Petsom, A. Petroleum Markers Synthesized from N-Alkylbenzene and Aniline Derivatives. Ind. Eng. Chem. Res. 2003, 42, 5054-5059. 